\title{
Kinetic Analysis on Decomposition of Pentaerythrityl Tetranitrate under Influence of Thiols
}

\author{
Marvel Reuben Suwitono ${ }^{1}$, R.E. Kartasasmita ${ }^{2}$, J.S. Pamudji ${ }^{2}$, and S. Ibrahim ${ }^{2}$ \\ ${ }^{1}$ Science Department, Universitas Advent Indonesia, \\ ${ }^{2}$ School of Pharmacy, Institut Teknologi Bandung, Indonesia \\ marvelreuben@yahoo.com
}

\begin{abstract}
Pentaerythrityl tetranitrate (PETN) is an organic nitrate compound used in the therapy as general vasodilator in the treatment of angina pectoris. PETN acts as prodrug for nitric oxide as active component. However, in clinical association, it is known a decreased ability of organic nitrate to do chemical reduction, which is metabolic tolerance, by upregulation in endogenic thiol system. It has been proved that supplementation of thiol is able to overcome tolerance to nitrate, nevertheless there is no comprehensive reports on interaction between organic nitrate and thiol compounds. The goal of this research is to study decomposition reaction kinetic of PETN under influence N-acetyl cysteine (NAC) and thiosalicylic acid (TSA) as thiol compounds, in the physiological condition. PETN as tracing compound were monitored by chromatographic system, with ratio of thiols used was equal, doubled, five times, and ten times folds. Data were analyzed kinetically by using math equation for reaction order 1, 2, and composite order 1 (equivalence). The result showed the linearity values on kinetic derivative, where correlation coefficient, $r$, is higher than $0.9(r \geq 0.9)$ for composite order 1 . The result will give scientific contribution about tolerance phenomena in organic nitrate, particularly in the role of thiol compound to overcome nitrate tolerance.
\end{abstract}

Keywords: Pentaerythrityl Tetranitrate, N-Acetyl Cystein, Thiosalicylic Acid, Kinetic Study

\section{INTRODUCTION}

Pentaerythrityl tetranitrate (PETN) is an long acting organic nitrate used in the therapy as anti-anginal medication caused by endothelial dysfunction in patients with coronary artery disease (Rutherford and Struthers, 2013). In animal models of diabetes and hypertension, PETN showed to improved vascular function. PETN has fewer side effects on the vascular, particularly in endothelial cell, than other nitrates like NTG (nitroglycerin), isosorbide-5mononitrate (ISMN), or isosorbide dinitrate (ISDN) (Steven et al., 2017).

Reducing compounds especially thiols, e.g. N-acetyl cysteine (NAC), play a role in the metabolism in activating organic nitrates to nitrite then liberate NO. Thiols acts as cofactors in the enzymatic bioactivation, and as reducing agents in the non-enzymatic via formation of nitro- and nitroso-thioles as precursors (Kartasasmita, 2002; Seeling and Lehmann, 2006). 
In the formation of nitric oxide (NO) as the endothelium-derived relaxing factor (EDRF), high potency of organic nitrates e.g. nitroglycerin (NTG), pentaerythrityl tetranitrate (PETN) and pentaerythrityl trinitrate (PETriN), were suggested to be bioactivated by mitrochondrial aldehyde dehydrogenase (ALDH-2), where cytochrome P450s is responsible for low potency of organic nitrate e.g. isosorbide dinitrate (ISDN), isosorbide mononitrate (ISMN), pentaerythrityl dinitrate (PEDN), and pentaerythrityl mononitrate (PEMN) (Münzel et al., 2011). Due to lack of technical aspect to detect direct NO, as well as for quantification of NO as primary product in the reaction of nitrate and thiol, thus, analysis is being focuses on its donor as indirect measurement of NO levels (Suwitono et al., 2011).

This study aims to study decomposition reaction kinetic of PETN under influence of thiol, e.g. N-acetyl cysteine (NAC) and thiosalicylic acid (TSA), in the physiological condition. High performance liquid chromatography (HPLC) method was used to quantify the amount of PETN change due to its reaction with thiols.

\section{METHODS}

\section{Chemicals}

Pentaerythrityl tetranitrate (PETN) (product of synthesis according to (Lange et al., 2009), thiosalicylic acid (TSA), N-acetyl-L-cysteine (NAC) (puriss, Fluka), NaCl, NaOH, H3PO4, $\mathrm{NaH} 2 \mathrm{PO} 4.12 \mathrm{H} 2 \mathrm{O}, \mathrm{Na} 2 \mathrm{HPO} 4$, sulfanilamide, N-(1-naphthyl)-ethylenediamine, $\mathrm{NaNO}$, $\mathrm{HCl}$, sulfanilic acid, acetic acid, alpha-naphthylamine. The chemicals used in this experiment were all analytical grade. All solvents were HPLC grade.

\section{Solution}

Solution of PETN $0.6 \mathrm{mM}$ were prepared by dissolving PETN with methanol, and added with water to have 50\% methanol solution of PETN. Then, the solution was filtered through 0.45 $\mu \mathrm{m}$ membrane filter. Solution of $6 \mathrm{mM}$ of TSA or NAC were prepared by dissolving TSA or NAC with small amount of methanol, and PBS solution was added to have concentrations ratios, which is equimolar, two, five and ten times of nitrates concentration.

Phosphate buffered saline (PBS) solution $(\mathrm{pH} \mathrm{7.4,} 10 \mathrm{mmol})$ were prepared by mixing $\mathrm{NaH} 2 \mathrm{PO} 4$ with $\mathrm{Na} 2 \mathrm{HPO} 4$ and $\mathrm{NaCl}$ in aqua bidestillata, to have phosphate strength of 10 mmol in physiological solution, and adjusted with either $10 \% \mathrm{NaOH}$ or $10 \%$ phosphoric acid solution to have $\mathrm{pH} 7.4$. 
Incubation

Decomposition of PETN was analyzed by adding PETN solution with equal volume to each concentration ratios of TSA and NAC in a well-stoppered glass apparatus. The mixture was then incubated in thermostated water bath at $37^{\circ} \mathrm{C}$. Solution of PETN was added equivolume with PBS solution, and treated as control. The aliquot was sampled $(n=3)$ at certain intervals of up to 240 minutes of incubation time, samples then freezed below $-20{ }^{\circ} \mathrm{C}$ until ready to be analyzed by HPLC, but not more than 1 week.

\section{HPLC Condition}

HPLC condition is optimized and validated to analyzed PETN. The HPLC system was Hitachi D-7000 with L-7400 UV/Vis detector, using RP C-18 column (phenomenex bond clone, 300 x $3.9 \mathrm{~mm}, 10 \mu \mathrm{m})$. Volume of HPLC sample loop was $20 \mu 1$. PETN was detected at $215 \mathrm{~nm}$ wavelength. Mobile phase was isocratic acetonitrile:water with ratio 1:1,1 $\mathrm{ml} / \mathrm{min}$

\section{RESULTS}

Validation of HPLC Condition

Validation of HPLC method was performed, and some parameter of HPLC condition to analyze PETN were obtained ( PETN showed significant linear regression along its concentration from $12.5 \mathrm{uM}$ up to $300 \mathrm{uM}$, (Figure 1) thus, this calibration equation could be used in conversion of concentration while detecting PETN.

Table 1). PETN was eluted at $12.7 \mathrm{~min}$, where TSA and NAC were around $2.6 \mathrm{~min}$. Examination of either TSA and NAC could not implement since newly product peak emerged at around TSA and NAC retention time, and piled up each other, therefore PETN used as tracing compound. PETN showed significant linear regression along its concentration from $12.5 \mathrm{uM}$ up to $300 \mathrm{uM}$, (Figure 1) thus, this calibration equation could be used in conversion of concentration while detecting PETN.

\section{Table 1. Validation Parameter of Chromatographic System for Detection of PETN}

\begin{tabular}{|l|l|}
\hline Parameter & Result \\
\hline Model & $\begin{array}{l}\text { Hitachi D-7000, Chromatography Data } \\
\text { Station Manager V 4.0, }\end{array}$ \\
\hline Wavelength & $215 \mathrm{~nm}$ \\
\hline Column & reverse phase C-18 (Phenomenex), 300 $\mathrm{mm}$ \\
\hline
\end{tabular}




\begin{tabular}{|l|l|}
\hline & x 3,9 $\mathrm{mm}$ i.d., particle size: $10 \mu \mathrm{m}$ \\
\hline Retention time & PETN =12.7 min., TSA \& NAC = 2.6 min., \\
\hline Selectivity & 21.2 \\
\hline Linearity & 0,999 \\
\hline Detection limit & $11,88 \mu \mathrm{M}$ \\
\hline Quantification limit & $33.57 \mu \mathrm{M}$ \\
\hline Accuracy & $0.70 \%($ of $<5 \%)$ \\
\hline Variation coef. & $2.82($ of $<5 \%)$ \\
\hline
\end{tabular}

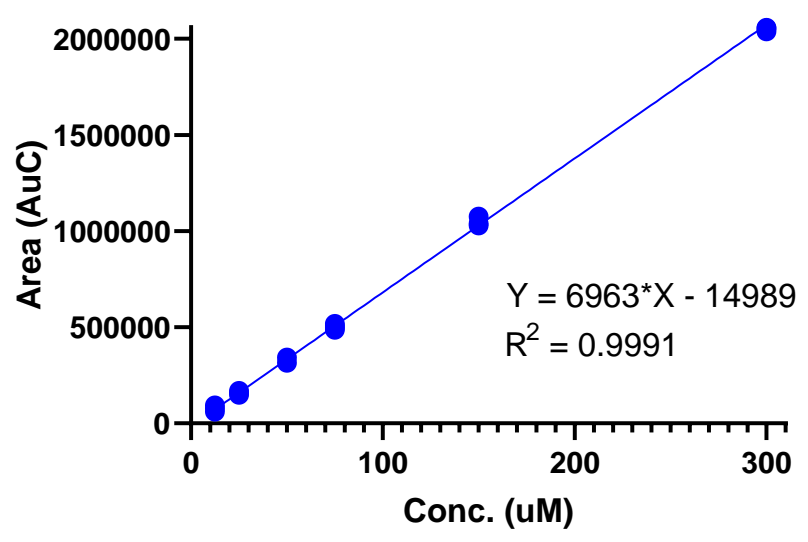

Figure 1. Calibration curve of PETN

Kinetic Decomposition

Decomposition of PETN were analyzed using validated HPLC method, under influence of TSA and NAC, in phosphate buffered-saline, mimic vascular buffer condition. Concentration of PETN showed extensive decreased in concentration while incubated with TSA and NAC, compared with its starting concentration, although at the end of incubation period there are still PETN remains (data is presented in Figure 2).

This condition applied for each variation ratios of TSA and NAC. Contrary, without neither TSA nor NAC, the profile showed stability by giving just slightly decreases of PETN. This result shows that TSA and NAC plays an important role in the degradation of PETN. 


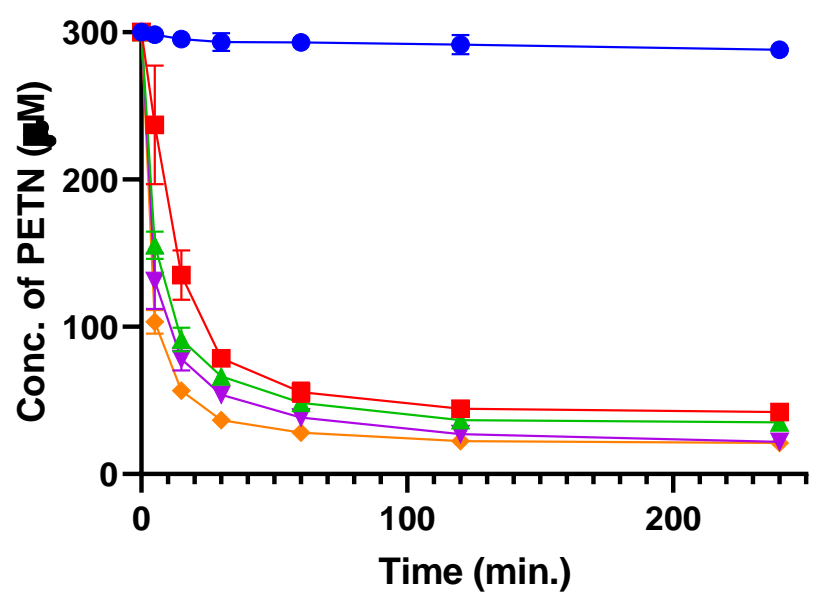

$$
\begin{gathered}
\text { Ratio of PETN:TSA } \\
\rightarrow \text { Ratio 1:0 } \\
\rightarrow-\text { Ratio 1:1 } \\
\rightarrow \text { Ratio 1:2 } \\
\rightarrow-\text { Ratio 1:5 } \\
\rightarrow-\text { Ratio 1:10 }
\end{gathered}
$$

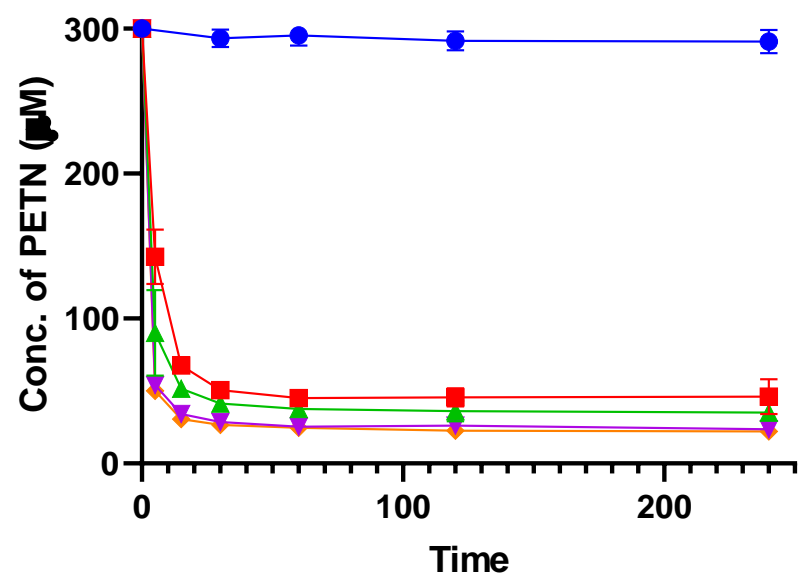

\section{Ratio of PETN:NAC}

$\rightarrow$ Ratio 1:0

$\rightarrow$ Ratio 1:1

$\rightarrow$ Ratio 1:2

$\rightarrow$ Ratio 1:5

$\rightarrow$ Ratio 1:10

Figure 2. Decomposition profile of PETN under influence of a) TSA, and b) NAC

\section{Kinetic Order}

Reaction order kinetic were calculated based on equilibrium condition, which is showing a nearly stable condition (no more significant reduction of PETN). Calculation of reaction rate is attempted with several kinetic models with curve fitting method, using several reaction order equations, which is narrowed to $1^{\text {st }}, 2^{\text {nd }}$ and composite $1^{\text {st }}$ order, showed in Table 2. Result of kinetic calculation from ordinate equation were shown in

Table 3, showing linearity significant value $r$, which is indicating acceptance of reaction order, with graphical plot of its mathematical derivation were shown in

Figure 3. 
Table 2. Reaction Order Equations

\begin{tabular}{|l|c|c|c|}
\hline $\begin{array}{c}\text { Reaction } \\
\text { order }\end{array}$ & $\begin{array}{c}\mathbf{1}^{\text {st }} \\
\text { order }\end{array}$ & $\mathbf{2}^{\text {nd }}$ order & $\begin{array}{c}\text { Composite } \mathbf{1}^{\text {st }} \\
\text { order }\end{array}$ \\
\hline $\begin{array}{c}\text { Ordinate } \\
\text { Equation }\end{array}$ & $\begin{array}{l}\ln \\
{[\mathrm{A}]}\end{array}$ & $\frac{x}{[A]_{0}\left([A]_{0}-x\right)}$ & $\ln \frac{\left\{[A]-[A]_{e q}\right\}}{\left\{[A]_{0}-[A]_{e q}\right\}}$ \\
\hline
\end{tabular}

Table 3. Recapitulation of Significance Value (r) of the Trend Line

\begin{tabular}{|l|c|c|c|c|}
\hline \multirow{2}{*}{ Reaction } & \multirow{2}{*}{ Molar rasio } & \multicolumn{3}{|c|}{ Significant value } \\
\cline { 3 - 5 } & & Order 1 & Order 2 & Composite \\
\hline PETN:NAC & $1: 1$ & 0,80 & 0,87 & 0,93 \\
\hline & $1: 2$ & 0,75 & 0,87 & 0,95 \\
\hline & $1: 5$ & 0,69 & 0,83 & 0,90 \\
\hline & $1: 10$ & 0,69 & 0,83 & 0,93 \\
\hline PETN:TSA & $1: 1$ & 0,95 & 0,99 & 0,99 \\
\hline & $1: 2$ & 0,89 & 0,97 & 0,96 \\
\hline & $1: 5$ & 0,88 & 0,98 & 0,95 \\
\hline & $1: 10$ & 0,85 & 0,96 & 0,96 \\
\hline
\end{tabular}
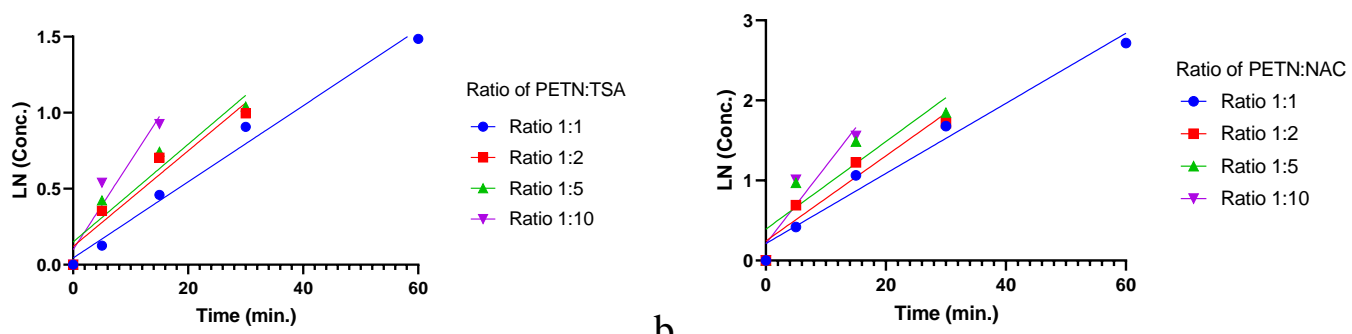

a.

Figure 3. Plot of mathematical derivatization of PETN concentration, with a) TSA, and b) NAC

Composite first order was chosen as best fit kinetic order for PETN decomposition scheme. This result could be explained as reaction of PETN with TSA and NAC were showed equilibrium at certain concentration of PETN (about 20\%). This might need further study to understand this phenomenon, including strength of phosphate buffer used, molar concentration of PETN and thiols used (TSA and NAC), different solvent, etc.

\section{DISCUSSION}

\section{Conclusion}


Analysis of PETN in this reaction scheme were performed using validated HPLC method. Chromatogram peak of PETN were completely separated from TSA and NAC, although simultaneous determination would not be possible due to the presence of products of reaction which are deleterious to NAC peak.

Result of this research confirmed that nitrate tolerance is occur when organic nitrate reacted with thiols, i.e. thiosalicylic acid (TSA) and n-acetyl-cystein (NAC). Nevertheless, the result showed that thiols plays important role in bioactivation of nitrates, as key on NO pathway.

\section{REFERENCES}

Kartasasmita, R.E., (2002). Perkembangan Obat Antiradang Bukan Steroid. Acta Pharmaceutica Indonesia. 27(4), 76-81.

Münzel, T., Daiber, A., \& Gori, T., (2011). Nitrate Therapy: New Aspects Concerning Molecular Action and Tolerance. Circulation, 123(19), 2132-2144. doi: https://doi.org/ 10.1161/CIRCULATIONAHA.110.981407

Seeling, A., \& Lehmann, J., (2006). NO-donors, part X [1]: Investigations on the stability of pentaerythrityl tetranitrate (PETN) by HPLC-chemoluminescence-N-detection (CLND) versus UV-detection in HPLC. Journal of Pharmaceutical and Biomedical Analysis. 40(5), 1131-1136. doi: https://doi.org/10.1016/j.jpba.2005.09.007

Steven, S., Oelze, M., Brandt, M., Ullmann, E., Kröller-Schön, S., Heeren, T., ... Daiber, A., (2017). Pentaerythritol Tetranitrate In Vivo Treatment Improves Oxidative Stress and Vascular Dysfunction by Suppression of Endothelin-1 Signaling in MonocrotalineInduced Pulmonary Hypertension. Oxidative Medicine and Cellular Longevity. 2017(1), 1-13. doi: https://doi.org/10.1155/2017/4353462

Suwitono, M.R., Kartasasmita, R.E., Pamudji, J.S., \& Ibrahim, S., 2011. The Role of Thiol on Degradation of Pentaerythityl Tetranitrate and Isosorbide Dinitrate. Journal of Applied Sciences. 11(24), 3854-3859. doi: https://doi.org/10.3923/jas.2011.3854.3859 